\title{
Mystery of bullet wound - a case study
}

\begin{abstract}
The case study relates to firearm suicide by a security personal and such incidents are very common among armed forces. Incident of firearm cases are often reported to forensic science laboratory for reconstruction to establish the crime. There are various reasons contributed for such incidents among security forces. It is observed, either head or chest is the most common site for self-inflicted firearm wound in almost all suicide cases. In one case, a security personal was on sentry duty with Indian Small Arms System (INSAS) rifle and committed suicide by his service firearm. The deceased was lying in a pool of blood along with firearm and one fired cartridge case near the body. The autopsy surgeon reported having entry wound on the back side situated in occipital bone $8 \mathrm{~cm}$ above cervical vertebra and exit wound over the forehead of the deceased. The observation was controversial since the presence of entry wound was at uncommon place showing absence of singeing, blackening, burning, tattooing and devoid of impression of the muzzle. In addition, it was also difficult for the shooter to fire from back side. Moreover, such type of incident has never been reported in literature in suicide cases by firearm. Further, bullet wound study along with spot analysis could confirm the entry wound to be on forehead and exit on the back. The mystery of origin of entry/exit wound could be established by wound ballistics study.
\end{abstract}

Keywords: suicide, bullet wound, service firearm, autopsy report, wound ballistics
Volume 4 Issue 3 - 2017

\author{
Ajitesh Pal, Pratihari HK \\ Tripura State Forensic Science Laboratory, India
}

Correspondence: Ajitesh Pal,Tripura State Forensic Science Laboratory, Agartala - 7990 I5, India,

Email ajiteshp@rediffmail.com

Received: January 31, 2017 | Published: April 03, 2017

\section{Introduction}

As per data of National Crime Records Bureau (NCRB), Government of India ${ }^{1}$ there is alarming suicide figure among different professionals and various means adopted for suicide in India. The poisonings considered to be highest category and use of firearm to be lowest since such tools are not easily available to common people. But suicide by security personnel in paramilitary forces is reported due to easy availability of firearm with them. In order to reconstruct the crime, physical evidence of shooting cases are referred for forensic examination along with autopsy study of the victim to establish the crime. The determination of suicide is supported not only by the presence of close range wound but also by crime scene study, case history of the victim along with autopsy report. The complete examination of the victim may also reveal evidence of self-intent on the hands of shoot deposit and blood spatter pattern. The suicides among security personnel are contributed for following reasons as reported in 'The Times of India'. '

1. Stress and over work among security personnel,

2. Poor working condition,

3. Denial of sanction of leave,

4. Depression after counter insurgency operation,

5. Lack of supervision and collection of intelligence from security personnel,

6. Intoxication leading to argument and resort to violence,

7. To take revenge on professional ground,

8. Quick action due to availability of firearm with them.

\section{Case report}

One rifleman working in battalion was assigned with sentry duty and all of a sudden firing sound was heard. On verification, it was found that the rifleman on sentry duty had committed suicide with his service firearm having bullet wounds one on the back side situated in occipital bone, $8 \mathrm{~cm}$ above cervical vertebra and the other wound over his forehead. The case was investigated and different physical evidence were collected to reconstruct the crime followed with autopsy study of the deceased.

\section{Material and methods}

In this case, the disputed service firearm, one fired cartridge case and a bullet fragment along with blood stain were collected from the crime spot for forensic examination. Subsequently, the scene of crime was also examined to reconstruct the incident and corroborate more facts in support of suicide and possible direction of firing. The seized cartridge case was examined by ballistics comparison microscope. The entry/exit wounds on the photograph were examined based on the wound ballistics.

\section{Results and discussion}

The direction of firing could be of great help in the reconstruction of event in this case. The systematic study of bullet holes (entry/ exit wounds) helped to establish the possible position of shooter and direction of firing. ${ }^{3}$ As per autopsy report, the entry wound was on the back side about $8 \mathrm{~cm}$ above the cervical bone and exit wound on the forehead. On close observation of the photograph of entry wound caused by contact firing, it was seen that the wound area was devoid of blackening/tattooing/ charring, also the absence of muzzle impression. As such, the possibility of having entry wound on back 
side was remote. Further, analysis of the photograph of exit wound (as per autopsy report) it was seen that the bullet injury is devastating and could cause when firing was made at the contact of hard skull. On contact or near contact firing range, the gas/flame produced by the combustion of the propellant could produce fatal injuries more than the injury by bullet. The skull being hard could not expand due to inadequate space for expansion of gas. As a result, large quantities of gas entering into the skull rebounded back and escaped through entry wound pulling out the soft brain matter. ${ }^{4}$ On spot analysis, it was found that one bullet mark was present on the roof of sentry post at acute angle with respect to the sitting position (cemented platform) of security personal. The position of bullet mark/dead body just after incident was suggestive of the muzzle end of rifle in upward direction (Figures 1-5).

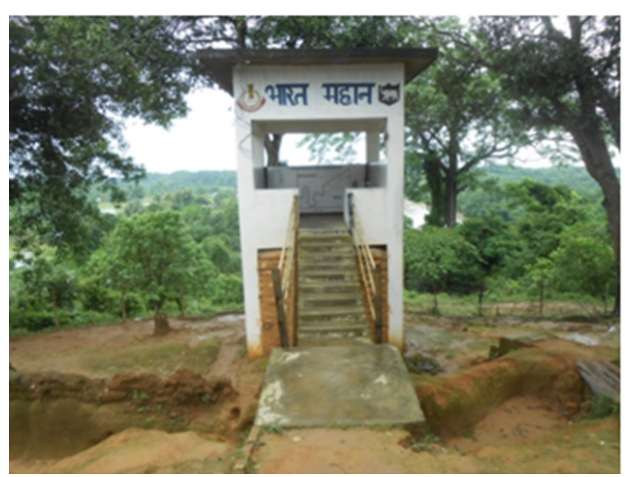

Figure I Sentry post located near international border.

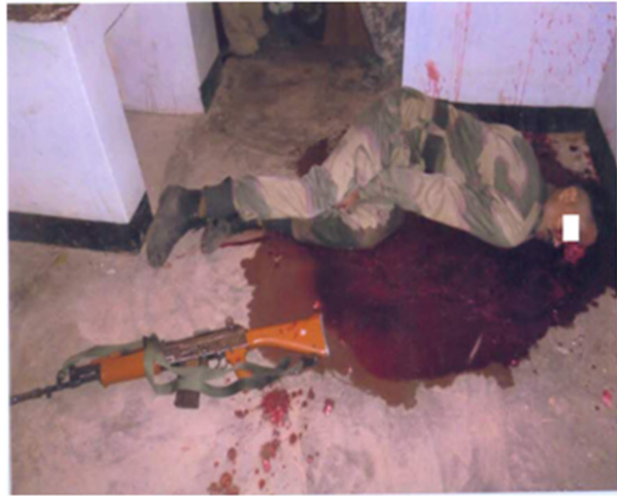

Figure 2 Deceased lying in a pool of blood with service firearm and fired cartridge case.

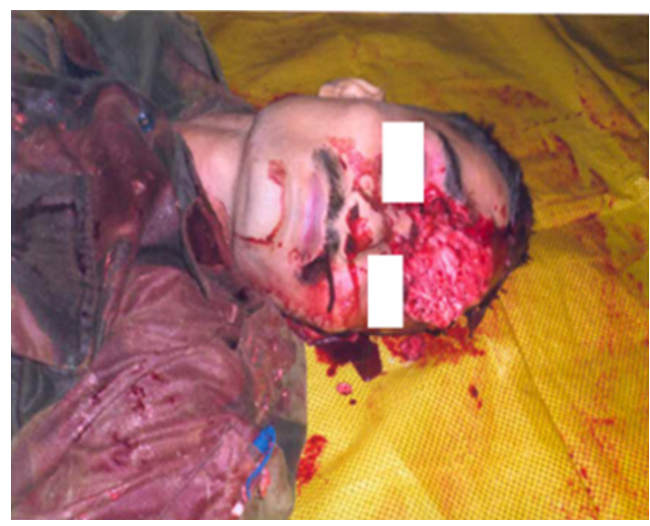

Figure 3 Exit wound on the forehead as per autopsy report.

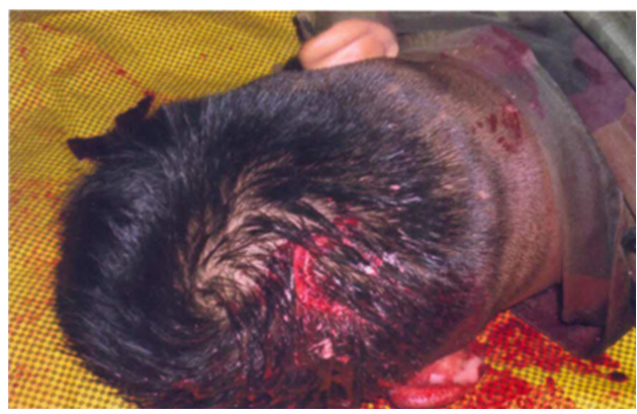

Figure 4 Entry wound on the back side as per autopsy report.

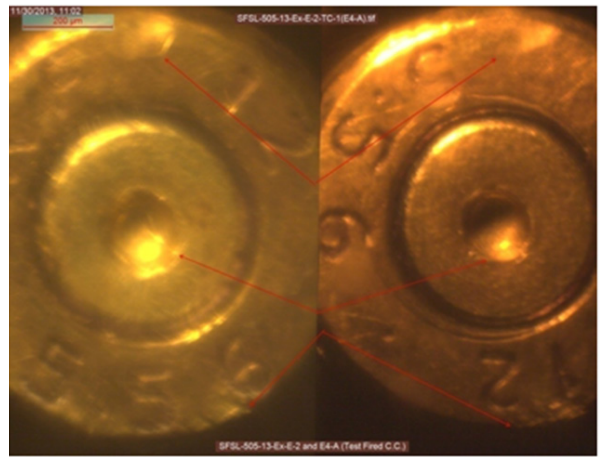

Figure 5 Comparison of test fired cartridge with crime cartridge case.

The other findings are mentioned below:

\section{Medico legal result}

Death is due to massive hemorrhage in brain due to firearm injury.

\section{Ballistics result}

The service firearm was recently fired as the nitrite test found positive. The recovered fired cartridge case was fired from the service firearm, confirmed through microscopic study.

\section{Toxicological result}

The viscera analysis ruled out the presence of common poison/ alcohol (Figure 6).
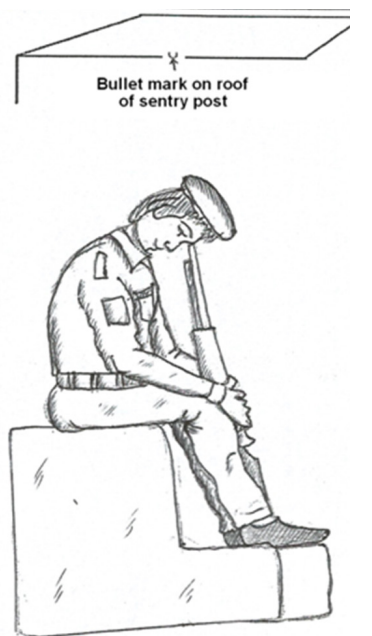

Figure 6 Possible position of the rifleman (shooter) reconstructed based on crime spot analysis \& wound ballistics. 


\section{Conclusion}

On the basis of wound ballistics study and crime spot analysis, the entry wound on the forehead and exit wound in the occipital bone on the back side of the deceased have been established. The recovered fired cartridge case was fired from the service firearm used by the security personal. The analysis of viscera ruled out the possibility of poisoning and confirmed the death due to firearm injury.

\section{Acknowledgments}

The authors are thankful to Dr. M.S. Rao, Ex-Chief Forensic Scientist, MHA, Govt of India for his guidance and also one of our staff Rajib Majumder for assistance.

\section{Conflicts of interest}

None.

\section{References}

1. Crime in India. National Crime Records Bureau Ministry of Home Affairs, India. 2013;1-474.

2. Rajat P. 597 military personnel have committed suicide in last 5 years, government says. The Times of India, India. 2014.

3. Sharma BR. Firearms in criminal investigation and trials. Forensic Science International. 1978;11(1):1-246.

4. Maio D. Gunshot Wounds. Practical Aspects of Firearms, Ballistics, and Forensic Techniques. 2nd edn. CRC Press. USA. 1999;1-401. 